\title{
ANÁLISE DE PRONTUÁRIOS DE PACIENTES ONCOLÓGICOS QUANTO AO MONITORAMENTO AUDITIVO
}

\author{
Analyzing medical records of oncologic patients \\ for audiological monitoring
}

Juliana Cunha da Costa ${ }^{(1)}$, Ceres Helena Buss ${ }^{(2)}$

\begin{abstract}
RESUMO
Objetivo: realizar uma análise retrospectiva dos prontuários de pacientes oncológicos quanto ao monitoramento auditivo. Métodos: foram utilizados os prontuários dos pacientes em atendimento durante os meses de setembro a novembro de 2006. A amostra constituiu-se de 25 prontuários de indivíduos com idade compreendida entre um e 53 anos. Foram analisados os dados quanto aos seguintes aspectos: idade, sexo, classificação do câncer, tempo de apresentação da doença, medicamentos utilizados, tempo de tratamento quimioterápico, doenças associadas, realização de avaliação otorrinolaringológica e audiológica. Resultados: verificou-se a falta de acompanhamento audiológico e otorrinolaringológico. A Vincristina foi o medicamento ototóxico mais utilizado. $O$ tipo de câncer de maior ocorrência no setor foi a Leucemia Linfocítica aguda. O tempo que os indivíduos apresentavam o câncer foi de aproximadamente 16 meses e o tempo de tratamento quimioterápico desses pacientes foi em torno de 11 meses. Observou-se, também, a presença de doenças associadas ao câncer nos indivíduos pesquisados. Conclusão: constatou-se a necessidade de se instalar programas de monitoramento auditivo nos setores que realizam quimioterapia em pacientes oncológicos, uma vez que este tratamento utiliza medicamentos ototóxicos em diversas patologias.
\end{abstract}

DESCRITORES: Monitoramento; Neoplasias; Quimioterapia; Perda Auditiva

\section{INTRODUÇÃO}

A integridade auditiva é de fundamental importância ao ser humano. Com a perda auditiva, a linguagem e a vida social do portador ficarão muito prejudicadas. Tendo em vista que o individuo é afetado diretamente pela perda auditiva, torna-se necessário identificar, diagnosticar e intervir precocemente, para proporcionar melhores condições a esse indivíduo.

O câncer (neoplasia, tumor maligno) é uma doença que vem crescendo em número de porta-

(1) Fonoaudióloga; Prefeitura Municipal de Toledo, PMT, Toledo, PR; Especialista em Fonoaudiologia pela Universidade Federal de Santa Maria; Especializanda em Saúde Pública da Universidade Estadual do Oeste do Paraná.

(2) Fonoaudióloga; Professora Adjunta do Departamento de Otorrinolaringologia e Fonoaudiologia da Universidade Federal de Santa Maria, UFSM, Santa Maria, RS; Doutora em Ciências dos Distúrbios da Comunicação Humana pela Universidade Federal de São Paulo / Escola Paulista de Medicina.

Conflito de interesse: INEXISTENTE dores, sendo a terceira maior causa de mortalidade no Brasil ${ }^{1}$. Nacionalmente, estima-se para o ano de 2009, 466.730 casos novos de câncer (CA) ${ }^{2}$. O CA é caracterizado por um grupo de células que crescem descontroladamente, capaz de invadir estruturas próximas e, ainda, se espalhar para diversas regiões do organismo. Dentre as formas de tratamento do CA está a quimioterapia que utiliza medicamentos para destruir as células tumorais. Cada vez que o paciente recebe o tratamento, são utilizadas várias drogas de classes diferentes ${ }^{1}$, dentre estas algumas são consideradas ototóxicas, as quais facilitam a instalação de perdas auditivas. Essas geram reações tóxicas às estruturas da orelha interna afetando o sistema auditivo e/ou vestibular. São consideradas drogas ototóxicas os aminoglicosídeos, a carboplatina, a cisplatina, entre outras $^{3}$.

Em pacientes expostos a drogas ototóxicas a monitorização auditiva é de fundamental importância, visto que resulta em uma detecção precoce das perdas auditivas induzidas por essas drogas, possi- 
bilitando a revisão do tratamento ou mesmo a escoIha de procedimentos alternativos, além de indentificar a progressão da lesão ${ }^{4,5}$. Caso estas medidas não sejam possíveis, a família e o paciente devem ser alertados para uma possível perda de audição, pois o prejuízo social da deficiência auditiva pode contribuir ainda mais para o isolamento do paciente portador de $\mathrm{CA}^{6}$.

Para a implementação de programas de monitoramento auditivo três aspectos devem ser considerados: prevenção e aconselhamento, monitoramento e reabilitação ${ }^{7}$. Atualmente a terapêutica contra o CA tem aumentado substancialmente a sobrevida das crianças com tumores malignos, sendo resultado de tratamentos mais efetivos como a quimioterapia combinada, a melhora no diagnóstico, nas técnicas cirúrgicas e radioterapêuticas, no uso combinado dos distintos recursos terapêuticos, no tratamento de suporte e melhoria da qualidade de vida ${ }^{4,8}$.

A ausência de dados sobre a ocorrência da realização de avaliações audiológicas durante e após o tratamento quimioterápico de pacientes oncológicos e a necessidade de se estabelecer rotinas no monitoramento da audição destes pacientes, bem como a escassez de trabalhos científicos que relacionem o CA com deficiência auditiva, principalmente no âmbito nacional, motivaram a realização deste estudo. Assim esta pesquisa teve como objetivo realizar uma análise retrospectiva dos prontuários de pacientes oncológicos quanto ao monitoramento auditivo, incluindo aspectos importantes a este monitoramento, como dados de identificação do paciente (idade e sexo), classificação do CA, tempo de apresentação da doença, medicamentos utilizados, tempo de tratamento quimioterápico, doenças associadas, realização de avaliação otorrinolaringológica e audiológica.

\section{MÉTODOS}

Neste trabalho, definiu-se por monitoramento auditivo, a realização das avaliações otorrinolaringológica e audiológica.

No período de setembro a novembro de 2006, foram atendidos no Centro de Tratamento da Criança com Câncer (CTCriaC) do setor de Oncologia do Hospital Universitário de Santa Maria, 109 pacientes com CA. Para a composição da amostra deste estudo foram selecionados os pacientes que realizaram o tratamento no período estudado e atenderam aos critérios de elegibilidade: realizar quimioterapia; utilizar, ou não, medicamentos ototóxicos; não realizar radioterapia e quimioterapia concomitantemente; ser paciente pediátrico ou adulto.
Desta forma, constituíram a amostra deste estudo, prontuários de 25 indivíduos que foram atendidos pelo setor e que haviam sido submetidos a tratamento quimioterápico durante o período de setembro a novembro de 2006. A idade destes 25 indivíduos foi compreendida entre um e 53 anos, sendo que, 15 eram do sexo masculino e 10 do sexo feminino.

A partir da amostra selecionada, realizou-se uma análise retrospectiva dos prontuários. A análise buscou dados como: identificação do paciente (idade e sexo), classificação do CA, tempo de diagnóstico do CA, medicamentos ototóxicos utilizados na quimioterapia (cisplatina, carboplatina, vincristina, bleomicina, neomicina, gentamicina, canamicina, polimixina, viomicina, amicacina, estreptomicina, paramomicina, tobramicina, e/ou diidroesptreptomicina) e medicamento otoprotetor (amifostina), tempo de tratamento quimioterápico, presença de doenças associadas, realização de avaliação otorrinolaringológica e /ou audiológica, com seus achados.

As informações coletadas tiveram a garantia do sigilo que assegura a privacidade e o anonimato dos sujeitos quanto aos dados confidenciais envolvidos na pesquisa.

Esta pesquisa contou com a aprovação do Comitê de Ética em Pesquisa da instituição, processo n'. 23081.015704/2006-23.

Pelas informações obtidas por meio dos prontuários, foi realizada uma análise estatística descritiva qualitativa, tabelas de freqüência e tabelas cruzadas, com o objetivo de verificar aspectos relevantes à pesquisa.

\section{RESULTADOS}

O estudo foi constituído de 25 prontuários de pacientes, sendo 10 (40\%) do sexo feminino e 15 $(60 \%)$ do sexo masculino. A análise dos resultados revelou que a idade dos pacientes variou de um a 53 anos, com média aritmética de 14 anos e um mês e desvio padrão de 10 anos e 11 meses. Verificando-se o tempo que os indivíduos apresentavam o diagnóstico do $\mathrm{CA}$, o tempo mínimo encontrado foi de 10 dias e o máximo de nove anos, sendo a média aritmética igual a 16 meses e seis dias, com desvio padrão de 26 meses e 18 dias.

Analisando-se o tempo que os indivíduos realizavam tratamento quimioterápico, o tempo mínimo encontrado foi de quatro dias e o máximo de cinco anos e dois meses, sendo a média aritmética igual a 11 meses e oito dias, com desvio padrão de 16 meses e 26 dias.

Em relação ao tipo de $C A$, constatou-se que, neste setor, o CA de maior incidência foi a Leucemia Linfocítica aguda, com sete indivíduos (28\%), 
seguida de três indivíduos (12\%) com Leucemia Mielóide Aguda e três indivíduos (12\%) com Linfoma B. de Grandes Células, os demais tipos de CA tiveram apenas uma ocorrência.

Quanto à presença de doenças concomitantemente ao CA, verificou-se que 22 indivíduos (88\%) apresentavam uma ou mais doenças associadas.

Considerando-se os 18 (72\%) indivíduos que fizeram uso de medicamentos ototóxicos, confrontou-se o tipo de CA com a utilização de medicamentos ototóxicos e realização de avaliação otorrinolaringológica, observando-se que o paciente com Leucemia Linfocítica Aguda, utilizou Vincristina, e foi o único indivíduo que fazia uso de medicamento ototóxico a realizar avaliação otorrinolaringológica (5,56\%).

Em outra comparação realizada, nos 18 (72\%) indivíduos, analisou-se a variável tipo de CA com a

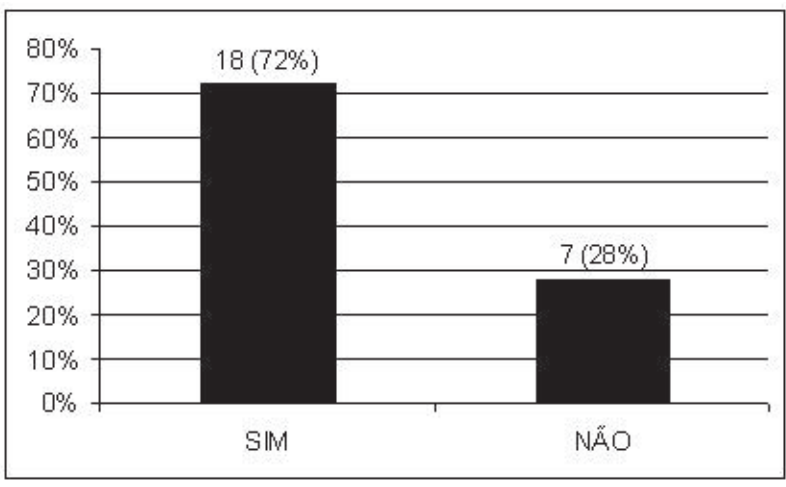

Figura 1 - Distribuição dos 25 indivíduos quanto à utilização de medicamentos ototóxicos

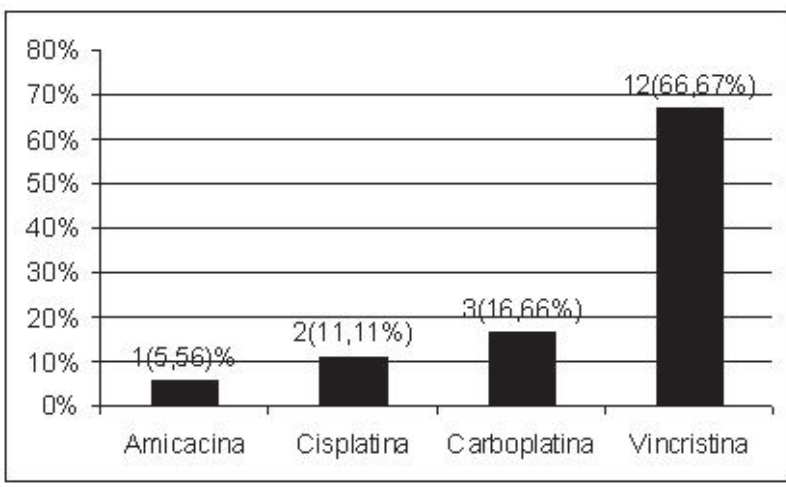

Figura 2 - Distribuição dos 18 indivíduos que fazem uso de medicamentos ototóxicos, quanto ao tipo de medicamento utilizado utilização de medicamentos ototóxicos e realização de avaliação audiológica. Percebendo-se que apenas um paciente $(5,56 \%)$, com Tumor de Células Germinativas Testicular Metástico, utilizando a cisplatina, realizou a avaliação audiológica.

Na Figura 1 está ilustrada a distribuição dos 25 indivíduos quanto à utilização de medicamentos ototóxicos. Na Figura 2, observa-se a distribuição dos 18 indivíduos que fazem uso de medicamentos ototóxicos, quanto ao tipo de medicamento utilizado. A Figura 3 demonstra a distribuição dos 25 indivíduos amostrados, quanto à variável realização de avaliação otorrinolaringológica. E a Figura 4 apresenta a distribuição do número de indivíduos, quanto à variável realização de avaliação audiológica, dos 25 indivíduos amostrados. Na Tabela 1 observa-se a comparação entre o tipo de CA e a utilização de medicamentos ototóxicos.

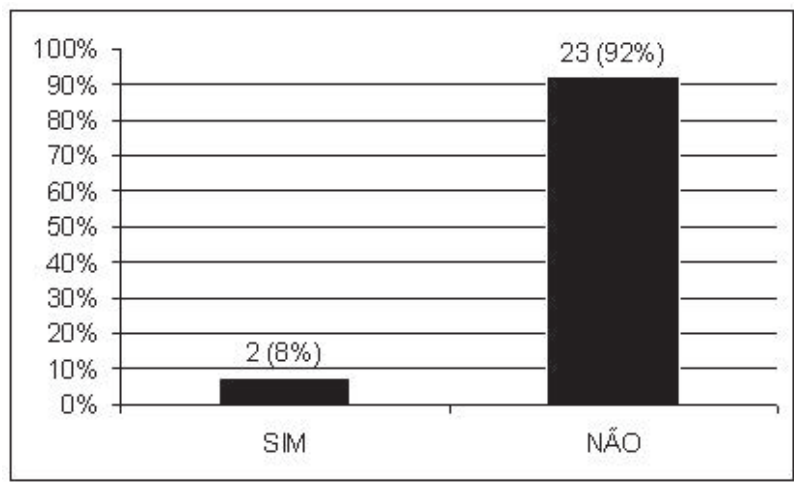

Figura 3 - Distribuição do número de indivíduos, quanto à variável realização de avaliação otorrinolaringológica, dos 25 indivíduos amostrados

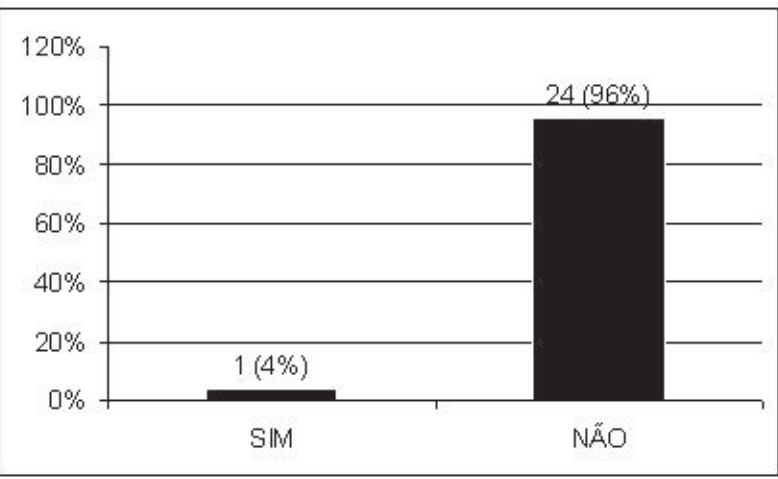

Figura 4 - Distribuição do número de indivíduos, quanto à variável realização de avaliação audiológica, dos 25 indivíduos amostrados 
Tabela 1 - Distribuição dos 18 indivíduos, quanto à variável tipo de CA com a utilização de medicamentos ototóxicos

\begin{tabular}{lccc}
\hline CÂNCER & $\begin{array}{c}\text { MEDICAMENTOS } \\
\text { OTOTÓXICOS }\end{array}$ & FREQUÊNCIA & PORCENTAGEM \\
\hline Leucemia Linfocítica Aguda & Vincristina & 5 & $27,78 \%$ \\
Linfoma de B. de Grandes Células & Vincristina & 2 & $11,11 \%$ \\
Hepatoblastoma & Cisplatina, & 1 & $5,56 \%$ \\
Linfoma Anaplásico de Grandes Células T. & Carboplatina & 1 & $5,56 \%$ \\
Linfoma de Burkitt & Vincristina & 1 & $5,56 \%$ \\
Leucemia Mielóide Aguda & Amicacina & 1 & $5,56 \%$ \\
Leucemia Mielóide Crônica & Vincristina & 1 & $5,56 \%$ \\
Linfoma não Hodgkin linfoblástico B. & Vincristina & 1 & $5,56 \%$ \\
Linfoma não Hodgkin linfoblástico T. & Vincristina & 1 & $5,56 \%$ \\
Neuroblastoma & Carboplatina, & 1 & $5,56 \%$ \\
Tumor de Células Germinativas Testicular & Vincristina & & $5,56 \%$ \\
Metástico & Cisplatina & 1 & $5,56 \%$ \\
Sarcoma Ewing & Carboplatina & 1 & $5,56 \%$ \\
Sarcoma Sinovial & Vincristina & 1 & $100 \%$ \\
\hline TOTAL & Vincristina & 18 & \\
\hline
\end{tabular}

\section{DISCUSSÃO}

Analisando os resultados deste estudo, observou-se que, em relação à prevalência do sexo, dos 25 indivíduos amostrados, 15 foram do sexo masculino e 10 do sexo feminino, verificando-se assim, maior ocorrência de indivíduos do sexo masculino. Estes achados estão de acordo com o estudo realizado entre os anos de 2003 e 2004, o qual foi constatado que $54,7 \%$ dos pacientes eram do sexo masculino $^{9}$; e em pesquisa de um centro de tratamento do CA pediátrico ${ }^{10}$, que verificou $52,1 \%$ de pacientes do sexo masculino que apresentavam CA; assim como outros estudos verificaram $67,2 \%{ }^{11} ; 63,2 \%{ }^{12}$; e $76,5 \%{ }^{13}$ pacientes do sexo masculino. Por outro lado, duas pesquisas tiveram a porcentagem do sexo feminino maior que do sexo masculino, com $55 \%{ }^{14}$ e $71 \%{ }^{15}$.

Quanto à variável idade, verificou-se que a idade mínima encontrada foi de um ano e a máxima de 59 anos, sendo a média aritmética igual a 14 anos e um mês, com desvio padrão de dez anos e 11 meses. Os resultados divergem dos estudos já realizados, que registraram a faixa etária mais freqüente entre 5 e 9 anos (38,3\%), seguida pela faixa de 10 anos e mais $(36,2 \%)^{6,10}$; e em registro de um centro de tratamento e pesquisa hospital do CA encontrou a mais alta taxa de ocorrência do CA nas crianças menores de cinco anos de idade ${ }^{16}$.

Em relação ao tipo de $\mathrm{CA}$, constatou-se que, neste setor, o CA de maior incidência foi a Leucemia Linfocítica Aguda, seguida de Leucemia Mielóide Aguda e Linfoma B. de Grandes Células, os demais tipos de CA tiveram apenas uma ocorrência. Resultados similares aos encontrados neste estudo foram observados por outros institutos que também constataram a leucemia como o diagnóstico mais frequente $(30 \%){ }^{17,18},(28 \%){ }^{19}$; e em pesquisa realizada por um centro de tratamento do $\mathrm{CA}$ pediátrico $(30,8 \%)^{6,10}$. E em outro estudo ${ }^{20}$, sobre o perfil epidemiológico do CA na rede pública em Porto Alegre/RS, a Leucemia Linfoblástica apresentou maior número de pacientes pediátricos e adolescentes por mês (37,5\%).

Analisando o tempo que os indivíduos apresentavam o diagnóstico do CA, o tempo mínimo encontrado foi de 10 dias e o máximo de nove anos, sendo a média aritmética igual a 16 meses e seis dias, com desvio padrão de 26 meses e 18 dias. Estes resultados informam, aproximadamente, o tempo em que os pacientes estavam em tratamento quimioterápico. $\mathrm{Na}$ literatura, esses dados foram considerados em estudo realizado em um centro de tratamento do CA pediátrico ${ }^{6,10}$, que observou que a idade ao diagnóstico variou de zero a 18 anos, 
sendo que menos da metade dos pacientes foram diagnosticados antes dos cinco anos (45,7\%); e em pesquisa realizada com crianças e adolescentes a qual também apresentou frequência semelhante nesta faixa etária (50\%) ${ }^{14}$. Entretanto, outro estudo apresentou a menor média de idade ao diagnóstico, sendo de 2 anos ${ }^{15}$.

Os achados quanto à utilização de medicamentos ototóxicos, constataram um número estatisticamente significante de indivíduos analisados que faziam uso destes medicamentos. Diferentes autores na literatura consultada definem a ototoxicidade, neste estudo considerou-se a ototoxicidade como sendo a tendência de certos agentes terapêuticos e outras substâncias químicas causarem prejuízo funcional e degeneração celular nos tecidos da orelha interna, levando à elevação dos limiares auditivos ${ }^{7}$. Torna-se extremamente importante a monitoração da audição, permitindo, desta forma, o diagnóstico precoce e a identificação da progressão da lesão.

Informações são muito importantes para o levantamento de indicadores de risco para a ocorrência de perda auditiva. Autores ${ }^{21}$ ressaltaram a importância de, previamente ao tratamento, questionar o paciente ou a seus familiares quanto ao uso recente de drogas ototóxicas e quanto à história familiar ou pessoal de alteração auditiva. Esses dados são importantes, uma vez que a concomitância de diferentes agentes tóxicos à orelha pode potencializar os efeitos sobre a audição, sendo que o uso destes é realidade no tratamento quimioterápico. Em relação aos 18 indivíduos que utilizavam medicamentos ototóxicos, o tipo de medicamento mais frequente foi o antineoplásico vincristina. Corroborando os achados deste estudo, pesquisa realizada encontrou a vincristina como o medicamento mais utilizado entre os pacientes $(58,5 \%)^{6}$. Afirmando o efeito ototóxico da vincristina, diferentes autores ${ }^{22-24}$ referiram a ototoxicidade deste antineoplásico.

Considerando o tempo pelo qual os indivíduos realizavam tratamento quimioterápico, o tempo mínimo encontrado foi de quatro dias e o máximo de cinco anos e dois meses, sendo a média aritmética igual a 11 meses e oito dias, com desvio padrão de 16 meses e 26 dias. Estes dados informaram o tempo que uma significante porcentagem dos pacientes deste estudo utilizaram medicamentos ototóxicos. Estas informações são importantes, pois como destacaram os autore ${ }^{4}$, a lesão auditiva que dependente de drogas ototóxicas apresenta caráter cumulativo.

Ainda de acordo com estes estudos ${ }^{4}$, a presença de alterações auditivas durante o uso de quimioterápicos está relacionada a diversos fatores, como administração da droga, localização do tumor, idade do paciente, medicamentos associados, susceptibilidade individual, função renal, irradiação craniana prévia, pré-existência de perda auditiva, dose cumulativa, dose total de tratamento.

Quanto à presença de doenças concomitantemente ao CA, verificou-se uma alta prevalência de indivíduos que apresentavam uma ou mais doenças associadas. Este fator foi analisado nesta pesquisa devido ao fato de algumas doenças ocasionarem risco a audição, o que excluiria, neste caso, a possibilidade de uma perda auditiva apenas pelo uso de drogas ototóxicas. Porém estas doenças não foram verificadas nos prontuários dos pacientes analisados. Na literatura examinada, observou-se achados em estudo realizado em centro de tratamento do CA pediátrico que constatou a queixa auditiva mais citada como sendo a otite $(22,3 \%)$, seguida de otalgia $(16,0 \%)$, tontura $(16,0 \%)$, dificuldade de audição (14,9\%) e infecção de vias aéreas superiores $(13,8 \%)^{6,10}$.

Diversos autores destacam fatores de risco para a ototoxicidade como a exposição a ruídos intensos; associação de drogas, como, por exemplo, uso concomitante de diuréticos; perdas auditivas prévias; problemas hepáticos ou renais; administração em crianças e recém-nascidos e pacientes em idade avançada; gravidez ${ }^{25}$; e dose cumulativa de cisplatina. Na presença de um desses fatores, o risco para perda auditiva é superior a $50 \%{ }^{25}$. Pesquisas relataram ainda, como fatores de risco para a ototoxicidade, a terapia prolongada, bacteremia, desidratação e febre. Já foi relatada tendência familiar a ototoxicidade ${ }^{26}$.

No presente estudo, observou-se que 23 dos 25 indivíduos não realizaram avaliação otorrinolaringológica no setor. Neste sentido, outros autores ${ }^{27}$ já enfatizaram a importância da avaliação otorrinolaringológica, referindo que a avaliação auditiva prévia ao tratamento e antes de cada dose da droga é fundamental para o diagnóstico precoce da perda auditiva. A possibilidade de alteração da quimioterapia, quando ocorrem perdas importantes e principalmente nos limiares da fala, deve ser discutida pelo otorrinolaringologista. Esta recomendação também é sugerida por outros autores ${ }^{21}$, que destacaram a necessidade do otorrinolaringologista e do fonoaudiólogo constituindo uma equipe multidisciplinar.

Os dados verificaram que apenas um dos indivíduos realizou a avaliação audiológica. Tal paciente apresentou audição dentro dos padrões de normalidade, entretanto não ficou esclarecido em que momento do tratamento a avaliação foi realizada. Demonstrando a necessidade de realizar avaliação audiológica em pacientes expostos a agentes 
quimioterápicos ou outros tipos de drogas ototóxicas, autores ${ }^{21}$ elucidaram a importância do monitoramento auditivo e relataram alguns aspectos determinantes na realização deste, como o fácil acesso do indivíduo às avaliações audiológicas que são realizadas dentro do próprio local do tratamento, ou ainda, a facilidade na locomoção para os centros especializados para a realização dessas avaliações, o que vem de encontro com os propósitos desta pesquisa. Outros autores referiram que as perdas auditivas são menores em centros nos quais é possível realizar a avaliação audiológica no próprio local de tratamento ${ }^{11}$.

A falta de avaliação audiológica é também salientada por estes autores ${ }^{6,10,21}$, que citam não ser rotina no Brasil, na grande maioria dos centros que atendem indivíduos em tratamento medicamentoso potencialmente ototóxico, como no caso dos centros de oncologia, realizar um monitoramento auditivo efetivo voltado aos pacientes que iniciam o tratamento do CA. Como principal dificuldade para realizar esse monitoramento destaca-se a ausência de um setor de audiologia que disponibilize os equipamentos necessários para esse fim. Além disso, o encaminhamento para a consulta com o otorrinolaringologista e para a avaliação audiológica ainda não é realidade.

Torna-se cada vez mais evidente a necessidade dos profissionais da área de Fonoaudiologia nos ambulatórios de oncologia, pois da mesma forma que o conhecimento sobre os diferentes tipos de quimioterápicos ou outros medicamentos ototóxicos vem aumentando, o diagnóstico precoce e, consequentemente, a cura do CA, estão cada vez mais presentes nestes ambulatórios, especialmente na oncologia pediátrica. Assim, o sucesso no tratamento do CA deve estar associado à preocupação com o bem-estar e a qualidade de vida do indivíduo em tratamento, o que inclui os cuidados com a audição ${ }^{21}$. Autores ${ }^{28}$ enfatizam que perdas auditivas quando não detectadas a tempo, podem trazer consequências em longo prazo.

O monitoramento deve ser realizado, sendo que o controle audiológico adequado consiste na avaliação auditiva anterior ao uso da droga ototóxica, controle periódico e reavaliação após o término da mesma ${ }^{4}$. Estas avaliações auditivas são importantes, pois podem determinar o grau e a progressão da ototoxicidade, principalmente nos casos de crianças em idade pré-linguística. Nestes casos, a perda auditiva representa um risco de atraso no desenvolvimento da fala, devido à dificuldade no reconhecimento de sons caracterizados por frequências altas ${ }^{29}$. Para monitorar a função auditiva, inúmeras são as técnicas que podem ser utilizadas durante a administração de drogas ototóxicas. Os exames utilizados para diagnosticar e prevenir os efeitos ototóxicos destas drogas são: a audiometria tonal liminar, potencial auditivo evocado de tronco cerebral, potencial endococlear e as emissões otoacústicas ${ }^{25}$.

Ao comparar o tipo de CA com a utilização de medicamentos ototóxicos, verificou-se que dos 18 indivíduos que fazem uso destes medicamentos, a Leucemia Linfocítica Aguda apresentou maior incidência de uso de ototóxicos, utilizando o antineoplásico vincristina. Não foram encontrados dados similares a estes na literatura, entretanto, estudo verificou o uso de cisplatina e/ou ifosfamida em pacientes com diagnóstico de leucemia linfocítica ${ }^{6}$.

Confrontando o tipo de CA com a utilização de medicamentos ototóxicos e realização de avaliação otorrinolaringológica, nos 18 indivíduos, observou-se que apenas um indivíduo que fazia uso de medicamento ototóxico realizou avaliação otorrinolaringológica. Este indivíduo apresentava Leucemia Linfocítica Aguda. Também não há na literatura consultada, achados comparando estes aspectos, todavia, em estudo sobre doenças sistêmicas em otorrinolaringologia do Hospital das Clínicas da FMUSP ${ }^{30}$, destacou que, como manifestações otorrinolaringológicas em pacientes com Leucemia, o osso temporal pode ser acometido pelas infiltrações leucêmicas no ouvido médio, mastóide e no ápice petroso, sendo que as manifestações podem ocorrer sob a forma de otite média secretora, otite média crônica, perda auditiva neurossensorial ou paralisia facial.

Em outra comparação realizada, analisou-se a variável tipo de CA com a utilização de medicamentos ototóxicos e realização de avaliação audiológica. Percebendo-se que apenas um indivíduo, portador de Tumor de Células Germinativas Testicular Metástico, que fazia uso de cisplatina, realizou a avaliação audiológica. Pesquisadores já referiam em outros estudos que o uso da cisplatina, droga conhecida por seu alto potencial ototóxico, foi feito por pacientes com diagnóstico de Tumor de Células Germinativas ${ }^{6,10}$, corroborando os achados deste trabalho. Em estudo realizado com 22 crianças e adolescentes diagnosticados com tumores sólidos e tratados com cisplatina, $73 \%$ apresentaram perda auditiva ${ }^{31}$.

Alguns dados deste estudo não foram analisados estatisticamente, porém são importantes, como a necessidade de se utilizar, no tratamento quimioterápico, medicamentos otoprotetores à ototoxicidade, como a amifostina e o extrato de ginkgo biloba, que já foram relatados na literatura. Em estudo realizado em 2003, com o extrato de ginkgo biloba e outro rea- 
lizado em 2005, com a amifostina, pesquisadores concluíram que ambos os medicamentos, por suas ações antioxidantes, atuam como otoprotetores a ototoxicidade pela cisplatina ${ }^{32,33}$. Entretanto alguns autores verificaram em seu estudo que a amifostina que a amifostina não protege contra a ototoxicidade causada pela combinação de cisplatina, etoposide e bleomicina, em pacientes com tumores de células germinativas ${ }^{6}$.

\section{CONCLUSÃO}

A análise dos resultados deste estudo retrospectivo dos prontuários de pacientes oncológicos quanto ao monitoramento auditivo, permitiu concluir que existe a falta de monitoramento auditivo, como também, a necessidade da realização de avaliações audiológicas e otorrinolaringológicas pela utilização de medicamentos ototóxicos.

\begin{abstract}
Purpose: to accomplish a retrospective analysis for medical records of oncologic patient for audiological monitoring. Methods: the patients' medical records in attendance were used from September to November, 2006. The sample constituted of 25 medical records of individuals with ages between 1 and 53-year old. The data were analyzed as for the following aspects: age, sex, cancer classification, time having the disease, used drugs, time of chemotherapy treatment, associated diseases, accomplishment of otorhinolaryngologic and audiologic evaluation. Results: we verified the lack of audiologic and otorhinolaryngologic care. Vincristina was the most used ototoxic drug. The type of cancer with larger occurrence in the section was the Acute Lymphocytic Leukemia. The time that the individuals had the cancer was of approximately 16 months and the time of chemotherapy treatment for the patient was about 11 months. We observed the presence of diseases associated to cancer in the studied individuals. Conclusion: we evidenced the need for installing audiological monitoring programs in the sectors that accomplish chemotherapy in oncologic patients, since that this treatment uses ototoxic drugs in several pathologies.
\end{abstract}

KEYWORDS: Monitoring; Neoplasms; Drug Therapy; Hearing Loss

\section{REFERÊNCIAS}

1. Associação Brasileira do Câncer [Site na Internet]. Disponível em: URL: http://www.daycare.com.br/ conteudo.aspx?idSecao=22\&idArea=2\&idConteud o=9. Acesso em 10 maio 2006.

2. Instituto Nacional do Câncer - INCA. Ministério da Saúde. Incidência de Câncer no Brasil [Site na Internet]. Disponível em: URL: http://www.inca.gov. br/estimativa/2008/index.asp?link=conteudo_view. asp\&ID=2. Acesso em 20 set 2008.

3. Schweitzer VG. Ototoxicity of chemotherapic agents. Otolaryngol Clin North Am. 1993; 26(5): 759-89.

4. Pedalini MEB, Cruz OLM, Bensadon RL. Aspectos audiológicos na radioterapia, quimioterapia e em tumores do ângulo ponto cerebelar. In: Angelis EC, Furia CLB, Mourão LF. A atuação da fonoaudiologia no câncer de cabeça e pescoço. São Paulo: Lovise; 2000. p. 273-82.

5. Jacob LCB. Monitoramento auditivo na ototoxicidade. In: Anais do 19Encontro Internacional de Audiologia 2004; Bauru, SP, Brasil.
6. Silva AM. Avaliação das perdas auditivas em crianças e adolescentes com câncer [dissertação]. São Paulo (SP): Faculdade de Saúde Pública, Universidade de São Paulo; 2006.

7. Arslan E, Orzan E, Santarelli R. Global problem of drug-induced hearing loss. Ann N Y Acad Sci. 1999; 884:1-14.

8. Veneziano DB, Latorre MRDO, Mirra AP, organizador. Incidência, mortalidade e sobrevida do câncer da infância no município de São Paulo. São Paulo: Registro de Câncer de São Paulo; 2004.

9. Fundação Oncocentro de São Paulo - FOSP. Boletim do registro hospitalar de câncer. São Paulo; 2004. [Site na Internet]. Disponível em: URL: http:// www.fosp.saude.sp.gov.br. Acesso em 17 maio 2006.

10. Silva AM, Latorre MRDO, Cristofani LM, Odone Filho $\mathrm{V}$. A prevalência de perdas auditivas em crianças e adolescentes com câncer. Rev Bras Otorrinolaringol. 2007; 73(5):608-14.

11. KnightKRG, KraemerDF, NeuweltEA. Ototoxicity in children receiving platinum chemotherapy: underestimating a commonly occurring toxicity that 
may influence academic and social development. J Clin Oncol. 2005; 23(34):8588-96.

12. Skowronska-Gardas A, Pedziwiatr K, Chojnacka $M$. Evaluation of quality of life in long-term survivors of paediatric brain stem tumors, treated with radiotherapy. Radiother Oncol. 2004; 70(3):269-73. 13. Rodriguez-Galindo C, Wofford M, Castleberry RP, Swanson GP, London WB, Fontanesi J, et al. Preradiation chemotherapy with methotrexate, cisplatin, 5-fluorouracil, and leucovorin for pediatric nasopharyngeal carcinoma. Cancer. 2005; 103(4): 850-7.

14. Li Y, Womer RB, Silber JH. Predicting cisplatin ototoxicity in children: the influence of age and the cumulative dose. Eur J Cancer. 2004; 40(16):2445-51.

15. Marina N, Chang KW, Malogolowkin M, London WB, Frazier AL, Womer RB, et al. Amifostine does not protect against the ototoxicity of high-dose cisplatin combined with etoposide and bleomycin in pediatric germ-cell tumors: a Children's Oncology Group study. Cancer. 2005; 104(4):841-7.

16. Centro de Tratamento e Pesquisa Hospital do Câncer A.C. Camargo. Registro hospitalar de câncer [Site na Internet]. Disponível em: URL: http://www. hcanc.org.br. Acesso em 20 dez 2006.

17. Hospital do Câncer A. C. Camargo. São Paulo: Departamento de Pediatria; 2005 [Site na Internet]. Disponível em: URL: http://www.hcanc.org.br/ dmeds/oncoped/ped3.html. Acesso em 06 out 2006.

18. Instituto de Tratamento do Câncer Infantil do Rio Grande do Sul - ICI/RS. Rio Grande do Sul; 2005. [Site na Internet]. Disponível em: URL: http://www. ici-rs.org.br/conteudo/ici/sop.htm. Acesso em 12 jul 2006.

19. Fundação Oncocentro de São Paulo - FOSP. São Paulo; 2006. [Site na Internet]. Disponível em: URL: http://www.fosp.saude.sp.gov.br. Acesso em 17 maio 2006.

20. Bittencourt R, Scaletzky A, Boehl JAR. Perfil epidemiológico do câncer na rede pública em Porto Alegre - RS. Rev Bras Cancerol. 2004; 50(2):95-101.

RECEBIDO EM: 26/08/2007

ACEITO EM: 12/01/2008

Endereço para correspondência:

Juliana Cunha da Costa

Rua Presidente José Linhares, 423

Toledo - PR

CEP:85902-350

E-mail: jccfono@yahoo.com.br
21. Jacob LCB, Aguiar FP, Tomiasi AA, Tschoeke SN, Bitencourt RF de. Monitoramento auditivo na ototoxicidade. Rev Bras Otorrinolaringol. 2006; 72(6):836-44.

22. Adams PW, Rose DP, Folkard J, Wynn V, Seed $M$, Strong R. Effect of pyridoxine hydrochloride (vitamin B6) upon depression associated with oral contraception. Lancet. 1973; 1:897-904.

23. Serafy A, Hashash M. The effect of vincristine on the neurological elements of the rabbit cochlea. J Laryngol Otol. 1981; 95(1):49-53.

24. Oliveira JA. Ototoxicidade. In: Costa SS, Cruz OLM, Oliveira JAA, editores. Otorrinolaringologia: princípios e prática. Porto Alegre: Artes Médicas; 1994. p. 215-21.

25. Hyppolito MA, Oliveira JAA. Ototoxicidade, otoproteção e autodefesa das células ciliadas da cóclea. Medicina. 2005 Jul-Dez; 38(3/4):279-89.

26. Bento RF, Miniti A, Marone SAM. Doenças do ouvido interno. In: Tratado de otologia. São Paulo: Edusp; 1998. p. 266-74.

27. Borges GC, Borges RHM, Baraúna GN, Lopes Filho O. Ototoxicidade causada pela cisplatina em crianças: estudo retrospectivo. Rev Bras Otorrinolaringol. 2001; 67(3):292-5.

28. Smits C, Swen SJ, Theo Goverts S, Moll AC, Imhof SM, Schouten-van-Meeteren AY. Assessment of hearing in very young children receiving carboplatin for retinoblastoma. Eur J Cancer. 2006; 42(4):492-500.

29. Skinner R. Best practice in assessing ototoxicity in children with cancer. Eur J Cancer. 2004; 40(16):2352-4.

30. Hospital das Clínicas da Fmusp. Doenças sistêmicas em otorrinolaringologia. Disponível em: URL: http://www.hcnet.usp.br/otorrino/sem47.doc. Acesso em 04 de abril 2006.

31. Skinner R, Pearson AD, Amineddine HA, Mathias $\mathrm{DB}$, Craft AW. Ototoxicity of cisplatinum in children and adolescents. Br J Cancer. 1990; 61(6):927-31. 32. Hyppolito MA, Oliveira JAA, Rossato M, Holanda F. Ototoxicidade da cisplatina e otoproteção pelo extrato de ginkgo biloba às células ciliadas externas: estudo anatômico e eletrofisiológico. Rev Bras Otorrinolaringol. 2003; 69(4):504-11.

33. Hyppolito MA, Oliveira AA, Lessa RM, Rossato M. Otoproteção da amifostina aos efeitos ototóxicos da cisplatina: estudo em cobaias albinas por emissões otoacústicas produtos de distorção e microscopia eletrônica de varredura. Rev Bras Otorrinolaringol. 2005; 71(3):268-73. 